\title{
Relationship between chemical composition and magnetic susceptibility in the alkaline volcanics from the Isparta area, SW Turkey
}

\author{
Ömer Elitok ${ }^{1, *}$, Züheyr KamaCi², M Nuri Dolmaz ${ }^{2}$, Kamil Yilmaz $^{1}$ and Meltem Şener ${ }^{2}$ \\ ${ }^{1}$ Süleyman Demirel University, Department of Geological Engineering, 32260 Isparta, Turkey. \\ ${ }^{2}$ Süleyman Demirel University, Department of Geophysical Engineering, 32260 Isparta, Turkey. \\ *e-mail: oelitok@mmf.sdu.edu.tr
}

\begin{abstract}
Potassium-rich volcanic rocks in the Isparta area (SW Turkey) consist mainly of older (Pliocene) volcanic rock suites (e.g., lamprophyre, basaltic trachyandesite, trachyandesite, trachyte) and younger (Quaternary) caldera forming lava dome/flows (e.g., tephriphonolite, trachyte) and pyroclastics (ash/pumice fall deposits and ignimbritic flows). The magnetic susceptibility $(K)$ was performed for both groups. The magnetic susceptibility value of the less evolved rocks characterized by $\mathrm{SiO}_{2}<57$ wt\% (e.g., basaltic trachyandesite, tephriphonolite, lamprophyric rocks) and having mostly mafic phenocrysts such as pyroxene, amphibole, and biotite-phlogopite is over 10 $\left(10^{-3}[\mathrm{SI}]\right)$. Fine to medium-grained and subhedral to anhedral opaque minerals are scattered especially in the matrix phase of the less evolved volcanic rocks. However, the $K$ value of the more evolved rocks (e.g., trachyte and trachyandesites) with $\mathrm{SiO}_{2}$ over $57 \mathrm{wt} \%$ vary between 0.1 and 28, but most of them below 10. SI values are negatively correlated with $\mathrm{SiO}_{2}, \mathrm{Na}_{2} \mathrm{O}$, but positively correlated with $\mathrm{Fe}_{2} \mathrm{O}_{3}, \mathrm{CaO}, \mathrm{MnO}, \mathrm{P}_{2} \mathrm{O}_{5}$ and $\mathrm{MgO}$ contents, suggesting inverse variation of SI with fractionation of potassic magma. That is to say that less evolved volcanic rocks have relatively higher magnetic susceptibility values in the volcanic suite. Fine to medium-grained and subhedral to anhedral Fe-Ti oxides are scattered mainly in the matrix phase of the less evolved volcanics, presumably cause the pronounced relatively higher magnetic susceptibility.
\end{abstract}

\section{Introduction}

The magnetic susceptibility $(K)$ is the degree of magnetization of a material in response to an external magnetic field (Hunt et al 1995; Oniku et al 2008) and defined as $K=J / H$, the ratio of the induced magnetization $(J)$ to the applied field $(H)$. The bulk susceptibility of rocks is controlled by content of ferromagnetic minerals (Fe-Ti oxides: e.g., pyrrhotite, ilmenite, magnetite, titanomagnetite) in various rock types (Balsley and Buddington 1960; Aydin et al 2007; Searle 2008). Therefore, the magnetic susceptibility of rocks is determined by their bulk chemistry and magnetic mineralogy (Aydin et al 2007). Magnitude of the magnetic susceptibility primarly reflects the abundance, nature (magnetite vs. silicates) and chemical composition (iron/magnesium ratio) of constituting minerals (Aydin et al 2007). Stress variation (Nishioka et al 2007) and petrofabrics of the rocks (Borradaile et al 1985, 1986; Grégoire et al 1998) play an important role on the anisotropy of magnetic susceptibility (AMS) of rocks.

In this paper, it is aimed (i) to present geochemical content of the volcanic rocks from the Isparta area (SW Turkey), and (ii) to discuss the relationship between magnetic susceptibility

Keywords. Magnetic susceptibility; geochemistry; potassic; volcanic; Isparta. 


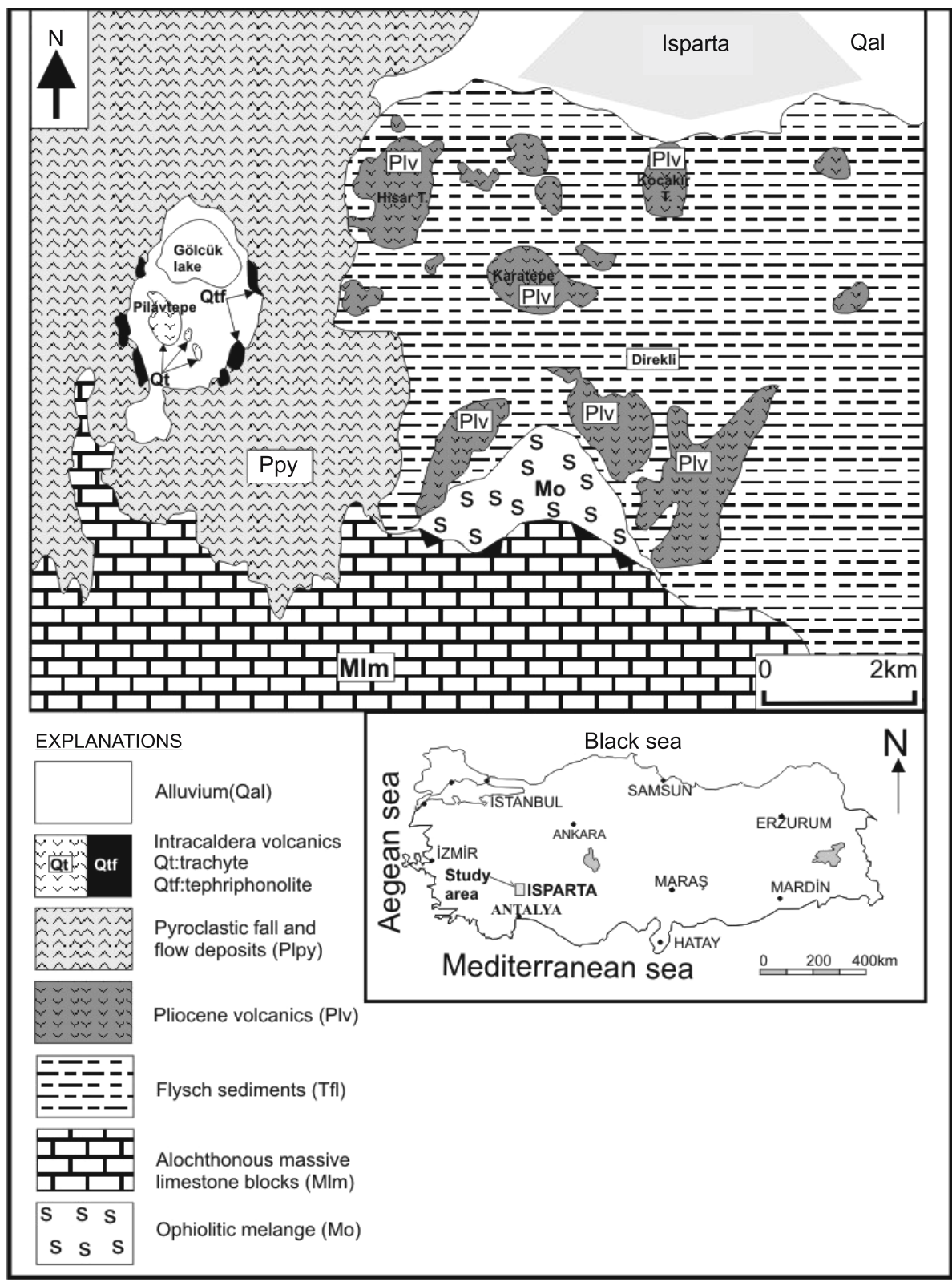

Figure 1. Geological map of the study area (modified from Kumral et al 2006).

and chemical composition of the volcanics using previous works.

\section{Geological setting}

The post-collisional potassium-rich Gölcük volcanics around Isparta take place at the apex of Isparta angle (SW Turkey). The study area is covered by autochthonous and allochthonous units which are intruded by Pliocene and Quaternary Gölcük volcanics and also overlain by pyroclastic ash/pumice fall and ignimbritic flow deposits. The Gölcük volcanics outcrop mainly in the south of Isparta settlement area, but small extrusions are also observed in the western and northern sides (figure 1). Platevoet et al (2008) separated the Gölcük volcanics into two major stages:

- older (Pliocene) lavas/domes, and

- younger (Quaternary) main volcano-forming stages which consist of three main eruptives cycles.

(a) Cycle I, represented by more than $200 \mathrm{~m}$ thick pyroclastic flow deposits occasionally separated by paleosoils and corresponding to caldera-forming ignimbritic eruptions.

(b) Cycle II, consisting of tephriphonolite lava dome-flows extruded throughout the caldera and currently found along the rim of the present crater. 
(c) Cycle III made up of tuff-ring deposits related to several phreatoplinian eruptions of a maar-type volcanic activity.

Elitok et al (2010) grouped all the Gölcük volcanics as: (i) extracaldera lavas, corresponding mainly to Pliocene activity; and (ii) intracaldera lavas (trachyte and tephriphonolite) and pyroclastics (ignimbrite flows and ash/pumice fall deposits) formed during the Quaternary. Extracaldera volcanics consist mainly of lamprophyre (minette), basaltic trachyandesite, trachyandesite, and trachyte (Elitok et al 2010). There is a close relationship between silica content and phenocryst types in the extracaldera volcanic rocks. In general, the phenocrysts in trachytes and trachyandesites with $\mathrm{SiO}_{2} \geq 57 \mathrm{wt} \%$ in the extracaldera volcanics are feldspars and mafic minerals (e.g., biotite, amphibole, and pyroxene), whereas the phenocrysts in trachyandesites and basaltic trachyandesites with $\mathrm{SiO}_{2}<57 \mathrm{wt} \%$ are mostly mafic with pyroxene, amphibole, and biotite-phlogopite (Elitok et al 2010).

\section{Petrology and magnetic susceptibility}

\subsection{Samples collection and analytical procedure}

Seven volcanic rock samples were collected from the field and analyzed for major, minor and trace elements. Geochemical analyses were conducted at the ACME Analytical Laboratories in Canada. Major and trace element contents were determined from fused $\mathrm{LiBO}_{2}$ discs by ICP-ES and ICP-MS, respectively, using five grams of sample powder. Geochemical data of 44 samples were taken from Elitok et al (2010). SI $\left(10^{-3}\right)$ measurements were performed using SM-30 magnetic susceptibility meter on the cubic specimens.

\subsection{Petrography}

The Gölcük volcanics have been divided into two subgroups: (i) extracaldera volcanics (older Pliocene lava protrusions) and (ii) younger Quaternary intracaldera lava domes (caldera-forming trachytic and tephriphonolitic lavas) and pyroclasti c deposits (ash/pumice fall deposits and ignimbritic flows). Extracaldera volcanics are represented mainly by lamprophyre, basaltic trachyandesite, trachyandesite, and trachyte. All petrographic descriptions are summarized in table 1. See Elitok et al (2010) for detailed petrographic descriptions. There is a close relationship between silica content and phenocryst types in this group. The phenocrysts in trachytes and trachyandesites with $\mathrm{SiO}_{2}>57 \mathrm{wt} \%$ are feldspars and mafic minerals (e.g., biotite, amphibole, and pyroxene), whereas the phenocrysts in trachyandesites and basaltic trachyandesites with $\mathrm{SiO}_{2}<57 \mathrm{wt} \%$ are mostly mafic with pyroxene, amphibole, and biotite-phlogopite.

\subsection{Geochemistry}

The chemical composition of major, minor element contents and the SI value of the volcanic rocks are presented in table 2. The Gölcük volcanics were classified using the total alkali $\left(\mathrm{K}_{2} \mathrm{O}\right.$ vs. $\left.\mathrm{Na}_{2} \mathrm{O}\right)$ wt\% vs. $\mathrm{SiO}_{2} \mathrm{wt} \%$ (TAS) diagram of Le Bas et al (1986) (figure 2). A positive correlation between $\mathrm{SiO}_{2}$ and total alkali content is observed among the extracaldera volcanics ranging from basaltic trachyandesites to trachytes, suggesting the important role of fractional crystallization in their evolution. In the TAS diagram, the Gölcük volcanics plot within the trachyte, trachyandesite, basaltic trachyandesite, tephriphonolite fields. Also, the lamprophyric rocks plot mainly within the basaltic trachyandesite and trachyandesite fields. Magnetic susceptibility values measured at these volcanic rocks range between 0 and $36\left(10^{-3}[\mathrm{SI}]\right)$ (table 2). Major element contents of the volcanic rocks plot against the SI to find out the relation between SI and geochemical features of the volcanic rocks and fractionation (figure 3). In this diagram, $\mathrm{SiO}_{2}, \mathrm{Na}_{2} \mathrm{O}$ show moderately negative (figures $3 \mathrm{a}, 3 \mathrm{~b}$ ), whereas $\mathrm{Fe}_{2} \mathrm{O}_{3}, \mathrm{CaO}, \mathrm{MnO}, \mathrm{P}_{2} \mathrm{O}_{5}$ and $\mathrm{MgO}$ display positive correlation with the magnetic susceptibility (SI), suggesting linear correlation with SI and fractionation (figures 3c, 3d, 3e, 3f, 3g). However, there is no relation between $\mathrm{TiO}_{2}, \mathrm{~K}_{2} \mathrm{O}$, and $\mathrm{Al}_{2} \mathrm{O}_{3}$ contents (figures $3 \mathrm{~h}, 3 \mathrm{l}, 3 \mathrm{j}$ ). As mentioned before, extracaldera volcanics with $\mathrm{SiO}_{2}<57 \mathrm{wt} \%$ are characterized by mostly mafic phenocrysts such as pyroxene, amphibole, and biotite-phlogopite. It is widely known that mafic rocks have a relatively high $\mathrm{Fe}-\mathrm{Ti}$ oxides content, up to $5 \%$, and their $\mathrm{Ti}$ content is high. Consistently, the magnetic susceptibility value of the volcanic rocks with $\mathrm{SiO}_{2}<57 \mathrm{wt} \%$ (e.g., basaltic trachyandesite, tephriphonolite, lamprophyric rocks) and having mostly mafic phenocrysts such as pyroxene, amphibole, and biotite-phlogopite is over $10\left(10^{-3}[\mathrm{SI}]\right)$. Fine to medium-grained and subhedral to anhedral opaque minerals are scattered especially in the matrix phase of the rocks. It is commonly known that magnetic susceptibility is normally controlled by a small group of iron-bearing minerals. Some minerals are much more magnetic than others, and some materials have more magnetic minerals than others. Magnetic susceptibility can help to identify the type of material and the amount of iron-bearing minerals it contains. Elitok et al (2010) reported 
Table 1. Petrographic features of the selected Gölcük volcanics.

\begin{tabular}{|c|c|c|c|c|c|c|c|c|c|c|c|c|c|c|c|c|c|}
\hline \multirow[b]{2}{*}{$\begin{array}{l}\text { Sample } \\
\text { no. }\end{array}$} & \multicolumn{3}{|c|}{ Matrix } & \multicolumn{8}{|c|}{ Phenocrystals } & \multicolumn{4}{|c|}{ Secondary minerals } & \multirow[b]{2}{*}{ Texture } & \multirow[b]{2}{*}{ Rock } \\
\hline & $\begin{array}{l}\text { mc士 } \\
\text { mcp } \\
\text { feld. }\end{array}$ & $\begin{array}{c}\text { micr. } \\
\text { mafic } \\
\text { minieral }\end{array}$ & $\begin{array}{c}\text { Cryist. } \\
\pm \text { volc } \\
\text { glass }\end{array}$ & san & plg & nep & ant & ol & $\operatorname{cpx}$ & amp & $\begin{array}{l}\text { bt } \pm \\
\text { phg }\end{array}$ & apt & sph & $\mathrm{zr}$ & opq & & \\
\hline Gl-2 & $\mathrm{X}$ & & & $\mathrm{X}$ & $\mathrm{X}$ & & & & $\mathrm{X}$ & & $\mathrm{X}$ & $\mathrm{X}$ & & & & $m p, f$ & $\mathrm{Ta}$ \\
\hline Gl-4 & $\mathrm{X}$ & & & $\mathrm{X}$ & $\mathrm{X}$ & & & & $\mathrm{X}$ & & $\mathrm{X}$ & $\mathrm{X}$ & & & $\mathrm{X}$ & $m p, f$ & $\mathrm{Ta}$ \\
\hline Gl-8 & & & $\mathrm{X}$ & $\mathrm{X}$ & $\mathrm{X}$ & & & & & & $\mathrm{X}$ & & $\mathrm{X}$ & & $\mathrm{X}$ & $\mathrm{p}$ & Bt-T \\
\hline Gl-13 & $\mathrm{X}$ & & $\mathrm{X}$ & $\mathrm{X}$ & $\mathrm{X}$ & & & & $\mathrm{X}$ & $\mathrm{X}$ & $\mathrm{X}$ & $\mathrm{X}$ & & & & $m p, f$ & $\mathrm{Ta}$ \\
\hline Gl-15 & $\mathrm{X}$ & & & $\mathrm{X}$ & $\mathrm{X}$ & & & & $\mathrm{X}$ & $\mathrm{X}$ & $\mathrm{X}$ & $\mathrm{X}$ & $\mathrm{X}$ & & $\mathrm{X}$ & $m p, f$ & $\mathrm{Ta}$ \\
\hline Gl-16 & $\mathrm{X}$ & & & $\mathrm{X}$ & $\mathrm{X}$ & & & & & $\mathrm{X}$ & $\mathrm{X}$ & & & & & $m p, f$ & $\mathrm{Ta}$ \\
\hline Gl-17 & $\mathrm{X}$ & $\mathrm{X}$ & $\mathrm{X}$ & & & & & & $\mathrm{X}$ & & $\mathrm{X}$ & $\mathrm{X}$ & & & $\mathrm{X}$ & $m p, f$ & $\mathrm{Ta}$ \\
\hline Gl-19 & $\mathrm{X}$ & & $\mathrm{X}$ & $\mathrm{X}$ & $\mathrm{X}$ & & & & $\mathrm{X}$ & & $\mathrm{X}$ & & & & $\mathrm{X}$ & $m p, f$ & Bt-Ta \\
\hline Gl-29 & $\mathrm{X}$ & & $\mathrm{X}$ & $\mathrm{X}$ & $\mathrm{X}$ & & & & & & $\mathrm{X}$ & & & & $\mathrm{X}$ & $m p, f$ & $\mathrm{Ta}$ \\
\hline Gl-32 & $\mathrm{X}$ & & & $\mathrm{X}$ & $\mathrm{X}$ & & & & $\mathrm{X}$ & $\mathrm{X}$ & $\mathrm{X}$ & $\mathrm{X}$ & $\mathrm{X}$ & & $\mathrm{X}$ & $\mathrm{p}$ & $\mathrm{Ta}$ \\
\hline Gl-39 & $\mathrm{X}$ & & & $\mathrm{X}$ & $\mathrm{X}$ & & & & $\mathrm{X}$ & $\mathrm{X}$ & $\mathrm{X}$ & $\mathrm{X}$ & & $\mathrm{X}$ & $\mathrm{X}$ & $m p, f$ & $\mathrm{Ta}$ \\
\hline Gl-42 & $\mathrm{X}$ & & & $\mathrm{X}$ & $\mathrm{X}$ & & & & & $\mathrm{X}$ & & & & & $\mathrm{X}$ & $m p, f$ & $\mathrm{~T}$ \\
\hline Gl-45 & & & $\mathrm{X}$ & $\mathrm{X}$ & $\mathrm{X}$ & & & & $\mathrm{X}$ & & $\mathrm{X}$ & $\mathrm{X}$ & $\mathrm{X}$ & & $\mathrm{X}$ & $\mathrm{p}$ & $\mathrm{Ta}$ \\
\hline Gl-47 & $\mathrm{X}$ & & & $\mathrm{X}$ & $\mathrm{X}$ & & & & $\mathrm{X}$ & & $\mathrm{X}$ & $\mathrm{X}$ & $\mathrm{X}$ & & $\mathrm{X}$ & $m p, f$ & $\mathrm{~T}$ \\
\hline Gl-50 & $\mathrm{X}$ & & & & & & & & $\mathrm{X}$ & & $\mathrm{X}$ & $\mathrm{X}$ & & & $\mathrm{X}$ & $m p, f$ & $\mathrm{Ta}$ \\
\hline Gl-57 & $\mathrm{X}$ & $\mathrm{X}$ & $\mathrm{X}$ & & & & & & $\mathrm{X}$ & & $\mathrm{X}$ & $\mathrm{X}$ & & & $\mathrm{X}$ & $\mathrm{mp}$ & $\mathrm{Ta}$ \\
\hline Gl-58 & $\mathrm{X}$ & & $\mathrm{X}$ & $\mathrm{X}$ & & & & $\mathrm{X}(?)$ & $\mathrm{X}$ & & $\mathrm{X}$ & $\mathrm{X}$ & & & & $\mathrm{p}$ & $\mathrm{T}$ \\
\hline Gl-59 & $\mathrm{X}$ & & & $\mathrm{X}$ & $\mathrm{X}$ & & & & $\mathrm{X}$ & $\mathrm{X}$ & $\mathrm{X}$ & $\mathrm{X}$ & & & & $m p, f$ & $\mathrm{Ta}$ \\
\hline Gl-60 & $\mathrm{X}$ & & & & & & & & $\mathrm{X}$ & $\mathrm{X}$ & $\mathrm{X}$ & $\mathrm{X}$ & & & $\mathrm{X}$ & $\mathrm{mp}$ & $\mathrm{T}$ \\
\hline Gl-61 & $\mathrm{X}$ & & $\mathrm{X}$ & & $\mathrm{X}$ & & & & $\mathrm{X}$ & $\mathrm{X}$ & $\mathrm{X}$ & $\mathrm{X}$ & & & & & $\mathrm{Ta}$ \\
\hline Gl-63 & & & $\mathrm{X}$ & $\mathrm{X}$ & $\mathrm{X}$ & & & & & & $\mathrm{X}$ & $\mathrm{X}$ & $\mathrm{X}$ & & $\mathrm{X}$ & $\mathrm{p}$ & $\mathrm{Ta}$ \\
\hline Gl-67 & $\mathrm{X}$ & $\mathrm{X}$ & & & & & & & $\mathrm{X}$ & & $\mathrm{X}$ & $\mathrm{X}$ & & & $\mathrm{X}$ & $m p, f$ & $\mathrm{Ta}$ \\
\hline Gl-69 & $\mathrm{X}$ & & & & $\mathrm{X}$ & & & & $\mathrm{X}$ & $\mathrm{X}$ & & $\mathrm{X}$ & & & $\mathrm{X}$ & $m p, f$ & $\mathrm{Ta}$ \\
\hline Gl-73 & $\mathrm{X}$ & & & $\mathrm{X}$ & $\mathrm{X}$ & & & & $\mathrm{X}$ & & & & $\mathrm{X}$ & & $\mathrm{X}$ & $m p, f$ & $\mathrm{~T}$ \\
\hline Gl-74 & & & $\mathrm{X}$ & $\mathrm{X}$ & $\mathrm{X}$ & & & & & & $\mathrm{X}$ & & & & $\mathrm{X}$ & $\mathrm{p}$ & $\mathrm{T}$ \\
\hline Gl-75 & $\mathrm{X}$ & & & & & & & & $\mathrm{X}$ & $\mathrm{X}$ & $\mathrm{X}$ & $\mathrm{X}$ & & & & $m p, f$ & $\mathrm{Ta}$ \\
\hline Gl-76 & $\mathrm{X}$ & & & & & & & & $\mathrm{X}$ & & $\mathrm{X}$ & $\mathrm{X}$ & & & $\mathrm{X}$ & $m p, f$ & $\mathrm{Ta}$ \\
\hline Gl-82 & $\mathrm{X}$ & & $\mathrm{X}$ & & & & & $\mathrm{X}$ & $\mathrm{X}$ & & & & & & $\mathrm{X}$ & $m p, f$ & $\mathrm{Ta}$ \\
\hline Gl-100 & & & $\mathrm{X}$ & $\mathrm{X}$ & $\mathrm{X}$ & & & & & $\mathrm{X}$ & & $\mathrm{X}$ & $\mathrm{X}$ & & $\mathrm{X}$ & $\mathrm{p}$ & $\mathrm{Ta}$ \\
\hline Gl-101 & $\mathrm{X}$ & & & & & & & & $\mathrm{X}$ & $\mathrm{X}$ & $\mathrm{X}$ & & $\mathrm{X}$ & & $\mathrm{X}$ & $m p, f$ & $\mathrm{Ta}$ \\
\hline Gl-105 & $\mathrm{X}$ & & $\mathrm{X}$ & $\mathrm{X}$ & $\mathrm{X}$ & & & & $\mathrm{X}$ & $\mathrm{X}$ & & & & & $\mathrm{X}$ & $\mathrm{p}$ & $\mathrm{Ta}$ \\
\hline Gl-111 & & & $\mathrm{X}$ & $\mathrm{X}$ & $\mathrm{X}$ & & & & $\mathrm{X}$ & $\mathrm{X}$ & $\mathrm{X}$ & $\mathrm{X}$ & & & & $m p, f$ & $\mathrm{Ta}$ \\
\hline Gl-112 & $\mathrm{X}$ & & $\mathrm{X}$ & & & & & & $\mathrm{X}$ & $\mathrm{X}$ & $\mathrm{X}$ & & & & $\mathrm{X}$ & $\mathrm{p}$ & $\mathrm{Ta}$ \\
\hline Gl-22 & $\mathrm{X}$ & & $\mathrm{X}$ & & & & & & $\mathrm{X}$ & & $\mathrm{X}$ & $\mathrm{X}$ & & & $\mathrm{X}$ & $m p, f$ & BTa \\
\hline Gl-48 & $\mathrm{X}$ & $\mathrm{X}$ & & & & & & & $\mathrm{X}$ & $\mathrm{X}$ & & & & & $\mathrm{X}$ & $m p, f$ & $\mathrm{BTa}$ \\
\hline Gl-55 & $\mathrm{X}$ & & & & & & & & $\mathrm{X}$ & & $\mathrm{X}$ & $\mathrm{X}$ & & & $\mathrm{X}$ & $m p, f$ & BTa \\
\hline Gl-81 & $\mathrm{X}$ & & $\mathrm{X}$ & & & & & & $\mathrm{X}$ & $\mathrm{X}$ & & $\mathrm{X}$ & $\mathrm{X}$ & & $\mathrm{X}$ & $m p, f$ & $\mathrm{BTa}$ \\
\hline Gl-96 & $\mathrm{X}$ & $\mathrm{X}$ & $\mathrm{X}$ & & & & & & $\mathrm{X}$ & & $\mathrm{X}$ & $\mathrm{X}$ & & & $\mathrm{X}$ & $\mathrm{p}$ & BTa \\
\hline Gl-88 & $\mathrm{X}$ & & $\mathrm{X}$ & $\mathrm{X}$ & $\mathrm{X}$ & $\mathrm{X}$ & & & $\mathrm{X}$ & & $\mathrm{X}$ & $\mathrm{X}$ & & & & $\mathrm{mp}$ & $\mathrm{Tp}$ \\
\hline Gl-97 & $\mathrm{X}$ & $\mathrm{X}$ & $\mathrm{X}$ & & & & & & $\mathrm{X}$ & & $\mathrm{X}$ & $\mathrm{X}$ & $\mathrm{X}$ & & $\mathrm{X}$ & $m p, f$ & $\mathrm{Tp}$ \\
\hline Gl-103 & $\mathrm{X}$ & & $\mathrm{X}$ & $\mathrm{X}$ & $\mathrm{X}$ & & & & $\mathrm{X}$ & & $\mathrm{X}$ & $\mathrm{X}$ & & & $\mathrm{X}$ & $\mathrm{p}$ & $\mathrm{Tp}$ \\
\hline JF-1 & $\mathrm{X}$ & $\mathrm{X}$ & $\mathrm{X}$ & $\mathrm{X}$ & $\mathrm{X}$ & $\mathrm{X}$ & & & $\mathrm{X}$ & & $\mathrm{X}$ & $\mathrm{X}$ & & & $\mathrm{X}$ & $\mathrm{p}$ & $\mathrm{Tp}$ \\
\hline JF-15 & $\mathrm{X}$ & $\mathrm{X}$ & $\mathrm{X}$ & & & & & & $\mathrm{X}$ & & & $\mathrm{X}$ & & & $\mathrm{X}$ & $\mathrm{p}$ & $\mathrm{Tp}$ \\
\hline JF-16 & $\mathrm{X}$ & $\mathrm{X}$ & $\mathrm{X}$ & & & & & & $\mathrm{X}$ & & $\mathrm{X}$ & $\mathrm{X}$ & & & $\mathrm{X}$ & $\mathrm{p}$ & $\mathrm{Tp}$ \\
\hline Gl-38 & $\mathrm{X}$ & $\mathrm{X}$ & $\mathrm{X}$ & & & & & & & & $\mathrm{X}$ & & & & & $\mathrm{mp}$ & Lmp \\
\hline Gl-40 & $\mathrm{X}$ & $\mathrm{X}$ & $\mathrm{X}$ & & & & & & $\mathrm{X}$ & & $\mathrm{X}$ & & & & $\mathrm{X}$ & $\mathrm{mp}$ & Lmp \\
\hline JF-8 & $\mathrm{X}$ & $\mathrm{X}$ & $\mathrm{X}$ & & & & & & $\mathrm{X}$ & $\mathrm{X}$ & $\mathrm{X}$ & & & & $\mathrm{X}$ & $\mathrm{p}$ & Lmp \\
\hline JF-9 & $\mathrm{X}$ & $\mathrm{X}$ & $\mathrm{X}$ & & & & & & $\mathrm{X}$ & & $\mathrm{X}$ & & & & $\mathrm{X}$ & $\mathrm{p}$ & Lmp \\
\hline JF-10 & $\mathrm{X}$ & $\mathrm{X}$ & $\mathrm{X}$ & & & & & & $\mathrm{X}$ & & $\mathrm{X}$ & & & & $\mathrm{X}$ & $\mathrm{p}$ & Lmp \\
\hline JF-23 & $\mathrm{X}$ & $\mathrm{X}$ & $\mathrm{X}$ & & & & & & $\mathrm{X}$ & & $\mathrm{X}$ & & & & $\mathrm{X}$ & $\mathrm{p}$ & Lmp \\
\hline
\end{tabular}

mc: microlite, mcp: microphenocrystal, feld: feldspat, san: sanidine, plg: plagioclase, nep: nepheline, ant: anorthoclase, ol: olivine, bt: biotite, phl: phlogopite, apt: apatite, sph: sphene, zr: zircon, opq: opaque, amp: amphibole, T: trachyte, Ta: trachyandesite, Tp: tephriphonolite, BTa: basaltic trachyandesite, Lmp: lamprophyre, p: porphyric, mp: microlitic porphyric, f: flow. 
Table 2. Representative major element contents and SI values of the Gölcük volcanics.

\begin{tabular}{|c|c|c|c|c|c|c|c|c|c|c|c|c|c|}
\hline Sample & Rock & $\mathrm{SiO}_{2}$ & $\mathrm{TiO}_{2}$ & $\mathrm{Al}_{2} \mathrm{O}_{3}$ & $\mathrm{Fe}_{2} \mathrm{O}_{3}$ & $\mathrm{MnO}$ & $\mathrm{MgO}$ & $\mathrm{CaO}$ & $\mathrm{K}_{2} \mathrm{O}$ & $\mathrm{Na}_{2} \mathrm{O}$ & $\mathrm{P}_{2} \mathrm{O}_{5}$ & LOI & $\mathrm{SI} \times 10^{-3}$ \\
\hline$* \mathrm{Gl}-13$ & $\mathrm{~T}-\mathrm{Ta}$ & 59.55 & 0.7 & 17.45 & 4.54 & 0.05 & 1.51 & 3.3 & 5.09 & 5.05 & 0.44 & 1.7 & 7 \\
\hline$*$ Gl-15 & $\mathrm{T}-\mathrm{Ta}$ & 58.17 & 0.52 & 18.61 & 3.89 & 0.09 & 1.51 & 3.69 & 5.82 & 4.25 & 0.28 & 2.9 & 17 \\
\hline$* \mathrm{Gl}-16$ & $\mathrm{~T}-\mathrm{Ta}$ & 58.88 & 0.61 & 19.81 & 2.97 & 0.03 & 1.14 & 3.67 & 5.61 & 4.95 & 0.38 & 1.6 & 0.2 \\
\hline$* \mathrm{Gl}-17$ & $\mathrm{~T}-\mathrm{Ta}$ & 56.94 & 0.79 & 16.03 & 4.74 & 0.07 & 3.32 & 5.25 & 6.51 & 3.49 & 0.56 & 1.8 & 14 \\
\hline *Gl-19 & $\mathrm{T}-\mathrm{Ta}$ & 64.21 & 0.48 & 17.02 & 3.36 & 0.06 & 0.9 & 2.6 & 5.24 & 4.81 & 0.22 & 0.8 & 9 \\
\hline$* \mathrm{Gl}-2$ & $\mathrm{~T}-\mathrm{Ta}$ & 60.4 & 0.69 & 16.5 & 4.71 & 0.05 & 2.28 & 4.39 & 5.21 & 4.38 & 0.38 & 0.6 & 1 \\
\hline$* \mathrm{Gl}-29$ & $\mathrm{~T}-\mathrm{Ta}$ & 66.09 & 0.41 & 17.09 & 1.37 & 0.005 & 0.49 & 1.51 & 5.04 & 5.24 & 0.19 & 2.2 & 0.1 \\
\hline *Gl-31 & $\mathrm{T}-\mathrm{Ta}$ & 56.79 & 0.53 & 17.05 & 4.42 & 0.1 & 1.47 & 5.36 & 5.76 & 4.26 & 0.29 & 3.4 & 7 \\
\hline$*$ Gl-39 & $\mathrm{T}-\mathrm{Ta}$ & 61.35 & 0.4 & 19.23 & 2.66 & 0.03 & 0.4 & 2 & 6.03 & 5.1 & 0.22 & 2.1 & 8 \\
\hline$* \mathrm{Gl}-4$ & $\mathrm{~T}-\mathrm{Ta}$ & 56.83 & 0.6 & 16.39 & 4.87 & 0.06 & 2.39 & 4.19 & 5.21 & 4.34 & 0.49 & 4.2 & 0.2 \\
\hline$* \mathrm{Gl}-42$ & $\mathrm{~T}-\mathrm{Ta}$ & 60.95 & 0.3 & 19.47 & 3.17 & 0.04 & 0.16 & 2.01 & 6.02 & 5.3 & 0.1 & 1.9 & 10 \\
\hline$* \mathrm{Gl}-45$ & $\mathrm{~T}-\mathrm{Ta}$ & 64.31 & 0.48 & 16.81 & 3.24 & 0.06 & 0.96 & 2.46 & 5.43 & 5.09 & 0.26 & 0.7 & 20 \\
\hline$* \mathrm{Gl}-47$ & $\mathrm{~T}-\mathrm{Ta}$ & 63.4 & 0.5 & 16.12 & 3.38 & 0.06 & 1.39 & 2.49 & 6.34 & 4.81 & 0.3 & 0.8 & 5 \\
\hline$* \mathrm{Gl}-50$ & $\mathrm{~T}-\mathrm{Ta}$ & 54.38 & 0.71 & 15.94 & 5.19 & 0.1 & 3.9 & 6.86 & 6.11 & 2.88 & 0.64 & 2.2 & 23 \\
\hline$* \mathrm{Gl}-57$ & $\mathrm{~T}-\mathrm{Ta}$ & 55.22 & 0.67 & 15.6 & 5.41 & 0.09 & 4.29 & 6.95 & 5.67 & 3.14 & 0.65 & 1.4 & 20 \\
\hline$* \mathrm{Gl}-58$ & $\mathrm{~T}-\mathrm{Ta}$ & 54.69 & 0.91 & 16.08 & 5.46 & 0.07 & 1.77 & 3.77 & 6.86 & 2.62 & 0.68 & 6.6 & 0.2 \\
\hline *Gl-59 & $\mathrm{T}-\mathrm{Ta}$ & 52.16 & 0.73 & 14.79 & 5.61 & 0.09 & 3.99 & 7.67 & 4.79 & 3.46 & 0.6 & 5.6 & 3 \\
\hline$* \mathrm{Gl}-60$ & $\mathrm{~T}-\mathrm{Ta}$ & 56.4 & 0.59 & 16.59 & 4.99 & 0.09 & 2.81 & 5.25 & 5.38 & 3.87 & 0.52 & 2.8 & 11.00 \\
\hline$*$ Gl-61 & $\mathrm{T}-\mathrm{Ta}$ & 57.11 & 0.59 & 17.95 & 4.8 & 0.09 & 2.7 & 4.96 & 4.87 & 4.72 & 0.64 & 1 & 15.00 \\
\hline$* \mathrm{Gl}-63$ & $\mathrm{~T}-\mathrm{Ta}$ & 65.55 & 0.5 & 15.36 & 3.59 & 0.05 & 0.98 & 1.83 & 4.88 & 4.06 & 0.27 & 2.8 & 0.50 \\
\hline$*$ Gl-67 & $\mathrm{T}-\mathrm{Ta}$ & 57.1 & 0.51 & 16.75 & 4.51 & 0.09 & 2.57 & 5.5 & 5.71 & 3.75 & 0.31 & 2.5 & 9.00 \\
\hline$*$ Gl-69 & $\mathrm{T}-\mathrm{Ta}$ & 53.19 & 0.62 & 16.8 & 5.45 & 0.11 & 2.11 & 6.72 & 5.75 & 2.67 & 0.49 & 5.4 & 18.00 \\
\hline$* \mathrm{Gl}-73$ & $\mathrm{~T}-\mathrm{Ta}$ & 63.93 & 0.21 & 19.23 & 1.94 & 0.04 & 0.07 & 1.17 & 6.46 & 6.05 & 0.05 & 0.8 & 7 \\
\hline$* \mathrm{Gl}-74$ & $\mathrm{~T}-\mathrm{Ta}$ & 64.88 & 0.4 & 17.25 & 2.52 & 0.02 & 0.61 & 0.9 & 5.59 & 5.95 & 0.23 & 1.2 & 8.00 \\
\hline$* \mathrm{Gl}-75$ & $\mathrm{~T}-\mathrm{Ta}$ & 55.71 & 0.65 & 17.48 & 5.37 & 0.1 & 2.83 & 5.9 & 5.62 & 4.06 & 0.55 & 1.1 & 19 \\
\hline$* \mathrm{Gl}-76$ & $\mathrm{~T}-\mathrm{Ta}$ & 52.54 & 0.68 & 17.89 & 6.1 & 0.11 & 2.86 & 6.53 & 5.73 & 2.54 & 0.62 & 3.5 & 28 \\
\hline$* \mathrm{Gl}-8$ & $\mathrm{~T}-\mathrm{Ta}$ & 66.31 & 0.53 & 16.99 & 2.38 & 0.02 & 0.56 & 1.9 & 5.33 & 4.68 & 0.29 & 0.8 & 2 \\
\hline$* \mathrm{Gl}-82$ & $\mathrm{~T}-\mathrm{Ta}$ & 57.52 & 0.9 & 14.86 & 5.56 & 0.09 & 4.47 & 6.88 & 3.41 & 4.12 & 0.55 & 1.4 & 16 \\
\hline$*$ Gl-83-B & $\mathrm{T}-\mathrm{Ta}$ & 55.93 & 0.47 & 18.59 & 4.31 & 0.11 & 1.18 & 4.31 & 5.68 & 4.87 & 0.29 & 3 & 17 \\
\hline *Gl-100 & $\mathrm{T}-\mathrm{Ta}$ & 64.4 & 0.43 & 17.35 & 3.29 & 0.07 & 0.74 & 1.6 & 4.98 & 5.31 & 0.25 & 1.2 & 0.5 \\
\hline *Gl-101 & $\mathrm{T}-\mathrm{Ta}$ & 54.5 & 0.65 & 17.44 & 4.45 & 0.08 & 3.24 & 5.93 & 4.87 & 3.51 & 0.99 & 3.7 & 17 \\
\hline *Gl-105 & $\mathrm{T}-\mathrm{Ta}$ & 66.25 & 0.41 & 16.63 & 2.39 & 0.04 & 0.92 & 3.3 & 4.07 & 5.04 & 0.13 & 0.4 & 3 \\
\hline *Gl-111 & $\mathrm{T}-\mathrm{Ta}$ & 61.12 & 0.53 & 17.89 & 4.18 & 0.06 & 0.71 & 3.09 & 5.24 & 4.89 & 0.32 & 1.6 & 7 \\
\hline *Gl-112 & $\mathrm{T}-\mathrm{Ta}$ & 57.81 & 0.6 & 17.23 & 4.56 & 0.1 & 2.07 & 2.91 & 6.13 & 3.7 & 0.41 & 4 & 13 \\
\hline$* \mathrm{Gl}-22$ & $\mathrm{BTa}$ & 50.38 & 0.7 & 17.33 & 6.72 & 0.13 & 3.08 & 7.63 & 5.5 & 2.28 & 0.67 & 4.5 & 36 \\
\hline$* \mathrm{Gl}-48$ & $\mathrm{BTa}$ & 51.29 & 0.82 & 16.09 & 5.59 & 0.11 & 3.57 & 9.12 & 5.74 & 2.22 & 0.69 & 4 & 20 \\
\hline$* \mathrm{Gl}-55$ & $\mathrm{BTa}$ & 51.92 & 0.78 & 16.51 & 6.01 & 0.11 & 4.52 & 8.25 & 4.82 & 2.74 & 0.64 & 2.8 & 20 \\
\hline$* \mathrm{Gl}-81$ & $\mathrm{BTa}$ & 50.6 & 0.65 & 16.15 & 5.79 & 0.12 & 3.44 & 9.06 & 4.56 & 3.24 & 0.61 & 5.1 & 19 \\
\hline *Gl-96 & BTa & 51.19 & 0.97 & 15.98 & 6.5 & 0.11 & 2.87 & 7.51 & 4.44 & 1.63 & 0.87 & 6.5 & 18 \\
\hline *Gl-103 & $\mathrm{Tf}$ & 51.94 & 0.73 & 16.21 & 6.27 & 0.11 & 3.75 & 8.1 & 5.39 & 4.58 & 0.81 & 1.2 & 26 \\
\hline$* \mathrm{Gl}-88$ & $\mathrm{Tf}$ & 54.1 & 0.68 & 17.09 & 5.56 & 0.1 & 3.06 & 6.62 & 5.75 & 3.67 & 0.63 & 2 & 13 \\
\hline *Gl-97 & $\mathrm{Tf}$ & 51.84 & 0.87 & 16.68 & 6.18 & 0.12 & 2.4 & 6.81 & 6.5 & 4.5 & 0.66 & 2.2 & 29 \\
\hline $\mathrm{JF}-1$ & $\mathrm{Tf}$ & 51.4 & 0.71 & 15.9 & 6.37 & 0.12 & 3.76 & 7.69 & 5.74 & 3.56 & 0.05 & 2.5 & 17 \\
\hline JF-15 & $\mathrm{Tf}$ & 50.28 & 0.73 & 15.23 & 6.66 & 0.13 & 4.23 & 8.78 & 5.61 & 4.11 & 0.99 & 1.7 & 13 \\
\hline JF-16 & $\mathrm{Tf}$ & 50.32 & 0.73 & 14.98 & 6.81 & 0.12 & 4.65 & 9.11 & 4.74 & 3.95 & 1.04 & 2 & 15 \\
\hline *Gl-38 & Lmp & 50.64 & 1.66 & 19.33 & 5.45 & 0.02 & 1.9 & 1.41 & 5.37 & 1.8 & 1.09 & 10.7 & 0.5 \\
\hline$* \mathrm{Gl}-40$ & Lmp & 50.18 & 1.41 & 16.34 & 6.17 & 0.07 & 4.33 & 6.46 & 5.06 & 1.84 & 1.05 & 6.5 & 18 \\
\hline JF-10 & Lmp & 47.9 & 1.53 & 17.3 & 7.29 & 0.1 & 4.9 & 7.71 & 3.48 & 2.28 & 1.12 & 5.4 & 13 \\
\hline JF-8 & Lmp & 53.65 & 0.92 & 16.29 & 5.04 & 0.07 & 4.31 & 6.75 & 4.45 & 2.81 & 0.71 & 4.2 & 15 \\
\hline JF-9 & Lmp & 48.52 & 1.04 & 15.91 & 7.5 & 0.13 & 6.21 & 10.9 & 3.66 & 3.37 & 0.74 & 0.8 & 21 \\
\hline $\mathrm{JF}-23$ & Lmp & 51.9 & 0.85 & 15.49 & 5.91 & 0.11 & 4.02 & 7.52 & 5.93 & 4.22 & 0.93 & 1.8 & 30 \\
\hline
\end{tabular}

(Geochemical data marked by '*' from Elitok et al 2010), T-Ta: trachyte-trachyandesite, BTa: basaltic trachyandesite, Tf: tephriphonolite, Lmp: lamprophyre. 


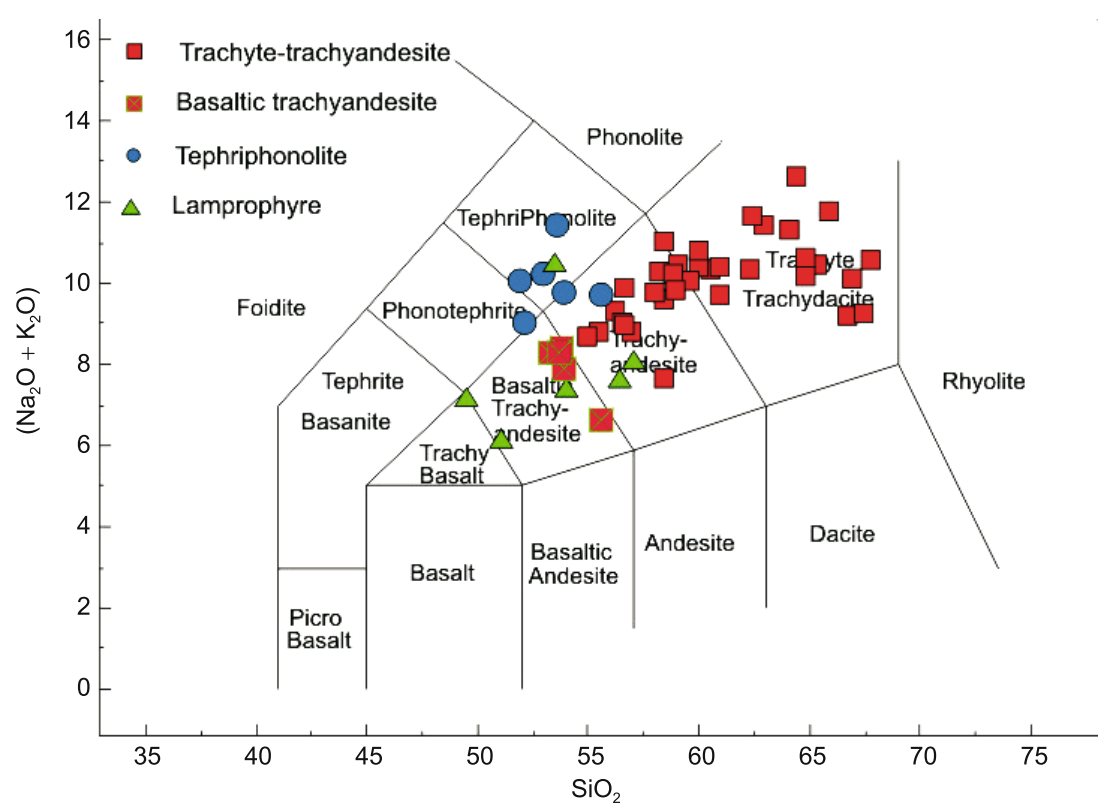

Figure 2. Total alkali vs. $\mathrm{SiO}_{2}$ diagram for the Gölcük volcanics. Classification scheme is from Le Bas et al (1986).

that early magnetite is a ubiquitous accessory constituent of all volcanic rock types in Isparta area. They also interpreted that biotite phenocrysts in basaltic trachyandesite samples $\left(X_{\mathrm{Mg}}\right.$ : $0.70-0.87$; Ti p.f.u.: $0.34-0.68)$ are more $\mathrm{Mg}$ - and Ti-rich than the biotite phenocrysts in the trachyandesites and can hence mainly be characterized as phlogopite.

\section{Discussion and conclusions}

The magnetic properties of minerals are mainly related mineralogical characteristics and nearly imperceptible differences, such as the replacement of a certain number of cations, lattice flaws, etc., can be reflected, highly amplified, in their magnetic characteristics. The most important ferromagnetic minerals are the Fe-Ti oxides and some Fe sulfides. Their susceptibility is variable: e.g., hematite $\left(1\left(10^{-3}[\mathrm{SI}]\right)\right)$ and magnetite can reach $0.107\left(10^{-3}[\mathrm{SI}]\right)$ (Lanza and Meloni 2006). The bulk magnetic susceptibility of rocks is the sum of the contributions from all Fe-bearing minerals (Thomson and Oldfield 1986; Verosub and Roberts 1995; Aydın et al 2007) and varies with concentration and composition of rock forming minerals which may include diamagnetic, paramagnetic or ferromagnetic. That is to say that magnetic susceptibility $(K)$ in different rocks is controlled by various mineral types and chemical composition: e.g., in granites by biotite (Oniku et al 2008), gabbros by the bulk $\mathrm{TiO}_{2}$ (Natland 2002), serpentinites by magnetite. However, extensive oxidation of magnetite may cause lower magnetic susceptibility (Nakamura and Borradaile 2004).
Positive values of $K$ imply that the induced magnetic field, $I$, is in the same direction as the inducing field, $H$, whereas negative values of $K$ imply that the induced magnetic field is in the opposite direction as the inducing field. Hunt et al (1995) interpreted that initial magnetic susceptibility is temperature dependent. They also stated that the susceptibility of paramagnetic materials is inversely proportional to absolute temperature, but the susceptibility of diamagnetic materials has no temperature dependence. Susceptibility and anisotropy of pre-existing ferrimagnetic minerals can be changed by heat that an increase of the susceptibility during heating is mainly due to growth of iron oxides (Henry et al 2003). A decrease could be often due to a transformation of these oxides, for example, by oxidation of magnetite to hematite (Henry et al 2003). Magnetic susceptibility $(K)$ may change with metamorphic grade from greenschist to granulite facies with depth (Nakamura and Borradaile 2004). Magnetic properties of rocks reflect the partitioning of iron between strongly magnetic oxides (and/or sulphides) and weakly magnetic phases such as silicates (Geuna et al 2008).

Plio-Quaternary volcanogenic rocks outcropping in the vicinity of Isparta (SW Turkey) are mainly in potassic character $\left(\mathrm{K}_{2} \mathrm{O}>\mathrm{Na}_{2} \mathrm{O}\right)$ and the volcanic activities are divided into two main stages:

- Pliocene volcanics dominated mainly by lava dome-flows, and

- Quaternary caldera-related volcanics (trachyte and tephriphonolite) and pyroclastics (ash/ pumice fall deposits and ignimbritic flows). 

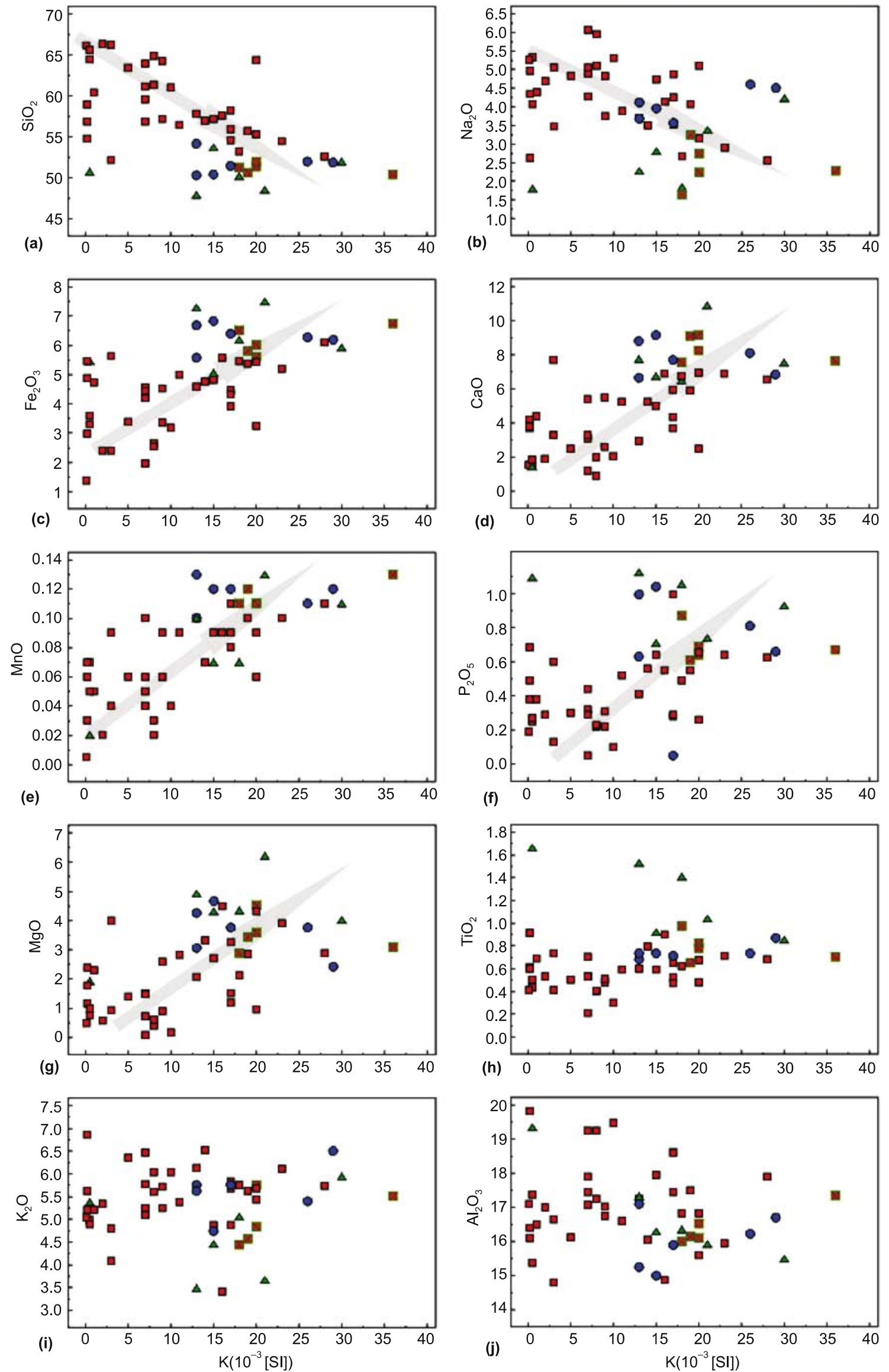

Figure 3. SI vs. major oxide variation diagrams for the Gölcük volcanics. Symbols as in figure 2. 
The Pliocene volcanics consist mainly of lamprophyre (minette), basaltic trachyandesite, trachyandesite and trachyte. SI values are negatively correlated with $\mathrm{SiO}_{2}, \mathrm{Na}_{2} \mathrm{O}$, but positively correlated with $\mathrm{Fe}_{2} \mathrm{O}_{3}, \mathrm{CaO}, \mathrm{MnO}, \mathrm{P}_{2} \mathrm{O}_{5}$ and $\mathrm{MgO}$ contents, suggesting inverse variation of SI with fractionation of potassic magma. That is to say that less evolved volcanic rocks have relatively higher magnetic susceptibility values. In petrographic investigations, it is mainly observed that fine to medium-grained and subhedral to anhedral Fe-Ti oxides are scattered mainly in the matrix phase of the volcanics with $\mathrm{SiO}_{2}<57 \mathrm{wt} \%$ and presumably cause the pronounced relatively higher magnetic susceptibility. Presumably, higher magnetic susceptibility values in the less evolved magmatic rocks reflect relatively high oxidation state of the magma, formed most probably in the subduction-related tectonic setting.

\section{Acknowledgements}

We would like to thank Dr R Jayangondaperumal and the anonymous referee for their constructive comments.

\section{References}

Aydın A, Feré E C and Aslan Z 2007 The magnetic susceptibility of granitic rocks as a proxy for geochemical composition: Example from the Saruhan granitods, NE Turkey; Tecotonophys. 441 85-95.

Balsley J R and Buddington A F 1960 Magnetic susceptibility anisotropy and fabric of some Adirondack Granites and orthogneisses; Am. J. Sci. Bradley 258-A 6-20.

Borradaile G, Mothersill J, Tarling D and Alford C 1986 Sources of magnetic susceptibility in a slate; Earth Planet. Sci. Lett. 76 336-340.

Elitok Ö, Özgür N, Drüppel K, Dilek Y, Platevoet B, Guillou H, Poisson A, Scaillet, S, Satır M, Siebel W, Bardintzeff J-M, Deniel C and Yılmaz K 2010 Origin and geodynamic evolution of late Cenozoic potassiumrich volcanism in the Isparta area, southwestern Turkey; Int. Geol. Rev. 52(4-6) 454-504.

Geuna S E, McEnroe S A, Robinson P and Escosteguy L D 2008 Magnetic petrology of the Devonian Achala
Batholith, Argentina: Titanohaematite as an indicator of highly oxidized magma during crystallization and cooling; Geophys. J. Int. 175 925-941.

Grégoire V, Darrozes J, Gaillot P and Nédélec A 1998 Magnetite grain shape fabric and distribution anisotropy vs. rock magnetic fabric: A three-dimensional case study; J. Struct. Geol. 20(7) 937-944.

Henry B, Jordanova D, Jordanova N, Souque C and Robion P 2003 Anisotropy of magnetic susceptibility of heated rocks; Tectonophys. 366 241-258.

Hunt C P, Moskowitz B M and Banerjee S K 1995 Magnetic properties of rocks and minerals; Rock Physics and Phase Relations, A Handbook of Physical Constants, AGU Reference Shelf 3.

Kumral K, Çoban H, Gedikoğlu A and Kılınç A 2006 Petrology and geochemistry of augite trachytes and porphyritic trachytes from the Gölcük volcanic region, Isparta, SW Turkey: A case study; J. Asian Earth Sci. 27 707-716.

Lanza R and Meloni A 2006 The Earth's Magnetism - An Introduction for Geologists (Berlin Heidelberg: SpringerVerlag), 267 p.

Le Bas M J, Le Maitre R W, Streckeisen A and Zanettin B 1986 A chemical classification of volcanic rocks based on the total alkali-silica diagram; J. Petrol. 27(3) 745-750.

Nakamura N and Borradaile G J 2004 Metamorphic control of magnetic susceptibility and magnetic fabrics: A 3-D projection; In: Magnetic Fabric Methods and Applications (eds) Martin-Hernandez F, Lüneburg C M, Aubourg C and Jackson M, Geol. Soc. London Spec. Publ. 238 61-68.

Natland J H 2002 Magnetic susceptibility as an index of the lithology and composition of gabbros, ODP Leg 176, Hole 735B, Southwest Indian Ridge; In: Natland J H, Dick H J B, Miller D J and Von Herzen R P (eds); Proc. ODP, Sci. Results 176 1-69.

Nishioka I, Funaki M and Sekine T 2007 Shock-induced anisotropy of magnetic susceptibility: Impact experiment on basaltic andesite; Earth Planets Space 59 e45-e48.

Oniku S A, Osazuwa I B and Meludu O C 2008 Preliminary report on magnetic susceptibility measurements on rocks within the Zaria granite batholith, Nigeria; Geofizika 25(2) 203-213.

Platevoet B, Scaillet S, Guillou H, Blamart D, Nomade S, Massault M, Poisson A, Elitok Ö, Özgür N, Yağmurlu F and Yılmaz K 2008 Pleistocene eruptive chronology of the Gölcük volcano, Isparta Angle, Turkey; Quaternaire 19(2) 147-156.

Searle R C 2008 Magnetic susceptibility as a tool for investigating igneous rocks - experience from IODP Expedition 304; Scientific Drilling 6 52-54, doi:10.2204/ iodp.sd.6.08.2008.

Thomson R and Oldfield F 1986 Environmental Magnetism (London: Allen and Unwin) $220 \mathrm{p}$.

Verosub K L and Roberts A P 1995 Environmental magnetism: Past, present and future; J. Geophys. Res. 100 2175-2192. 